\title{
Circulating cell-free mtDNA content is associated with outcome of HCC patients receiving TACE combined with traditional Chinese medicine treatment
}

\section{Guanlin Zhou}

Gannan Medical University

\section{Ying Li}

Gannan Medical University

\section{Shicheng Li}

Gannan Medical University

Hongxia Liu

Gannan Medical University

\section{Fei Xu}

Gannan Medical University

Xiaohuan Lai

Gannan Medical University

Qiong Zhang

Gannan Medical University

Jingxiang Xu ( $\nabla$ xunov10@sina.com)

Gannan Medical University

\section{Shaogui Wan}

Gannan Medical University

\section{Research}

Keywords: Hepatocellular carcinoma, mitochondrial DNA, TACE, traditional Chinese medicine, prognosis

Posted Date: May 21st, 2021

DOl: https://doi.org/10.21203/rs.3.rs-540053/v1

License: (c) (1) This work is licensed under a Creative Commons Attribution 4.0 International License. Read Full License 


\section{Abstract}

Objectives: Hepatocellular carcinoma (HCC) accounts for $70 \%-85 \%$ of liver cancer, and about $85 \%$ of HCC are hepatitis B virus-related hepatocellular carcinoma (HBV-HCC) in china. Most patients are already in the middle or late stages of the disease at the time of diagnosis, trans-hepatic arterial chemoembolization (TACE) combine with traditional Chinese medicine (TCM) have been reported as an effective treatment, and effective prognostic molecular markers are helpful to predict therapeutic efficacy. In this study, we aim to explore whether circulating cell-free mitochondrial DNA (ccf-mtDNA) content is associated with the outcome for HCC patients receiving TACE combine with TCM treatment.

Method: Retrospective analysis was conducted in a cohort with $141 \mathrm{HBV}-\mathrm{HCC}$ patients. Univariate and multivariate analysis was conducted with Cox proportional risk regression model to explore the correlation between ccf-mtDNA content and patient prognosis. Kaplan-Meier method was used to draw the survival curve of ccf-mtDNA content and the survival prognosis of patients.

Results: (1) high content of serum ccf-mtDNA is an independent risk factor for the prognosis of HBV-HCC patients treated with TACE combined with TCM adjuvant therapy $(\mathrm{HR}=4.010,95 \% \mathrm{IC}=1.252-12.844, \mathrm{P}=$ 0.019). (2) K-M survival analysis showed that patients with high ccf-mtDNA content had poor prognosis (Log Rank P=0.027).

Conclusions: Our findings suggest that ccf-mtDNA is a potential novel non-invasive biomarker for prognosis of HCC patients receiving TACE combine with TCM treatment.

\section{Introduction}

Hepatocellular carcinoma (HCC) is a second leading cause of cancer death, and Chronic hepatitis B virus (HBV) infection accounts for at least $50 \%$ cases of HCC worldwide ${ }^{1}$. Notably, HBV-related HCC accounts for about $85 \%$ of $\mathrm{HCC}$ cases in $\mathrm{China}^{2}$. The vast majority of HCC patients are diagnosed in middle or later stages, and curative treatments like surgical resection are not suitable for this patients ${ }^{3}$. Trans-arterial chemoembolization (TACE) is the standard of care for patients with intermediate HCC according to The barcelona clinic liver cancer (BCLC) staging system, and studies indicate TACE combined with traditional Chinese medicine (TCM) regimen displayed high efficacy in treating advanced $\mathrm{HCC}^{4}$.

Mitochondria are ubquitous eukaryotic cell organelles which playing an important role in energy production, cell proliferation as well as apoptosis ${ }^{5}$. The circular genome of mitochondrial (mtDNA) encodes for proteins essential in the oxidative phosphorylation system and the tRNA and rRNA molecules of the mitochondrial translation apparatus. Mitochondrial dysfunction plays an important role in the occurrence and development of liver cancer ${ }^{6,7}$, and when mitochondrial dysfunction causes mtDNA damage, mtDNA fragments can escape from the matrix and enter the cytosol or systemic circulation ${ }^{8}$. And changes in mtDNA content of tumor specimens have been reported ${ }^{9}$, the alteration of $\mathrm{mtDNA}$ content or sequence mutations has evitable relationship with oncogenesis and becomes potential biomarkers for 
certain types of cancers ${ }^{10}$, Alterations of mtDNA are generally accepted as independent biomarker for predicting risk and prognosis in various cancers, Bao et al found mtDNA copy number are associated with overall survival in hepatocellular carcinoma patients treated with transarterial chemoembolization ${ }^{11}$. mtDNA also may be released at low levels into the circulation from mitochondria under cellular stress, resulting in circulating cell-free mtDNA (ccf-mtDNA) detectable in plasma ${ }^{12}$. Recently circulating mtDNA have been targeted due to its specific and unique characteristics, which could potentially used as a novel biomarker for diagnostic and prognostic of cancer $^{13,14}$. Studies on the correlation between ccf-mtDNA content and the risk of HCC showed that the serum ccf-mtDNA content of HBV-HCC patients was significantly lower than that of the control group (non-HCC patients with HBV infection); and HBV infection with higher mtDNA content compared to patients with lower mtDNA content have a significantly higher risk of developing $\mathrm{HCC}^{13}$. However, the association of mtDNA content with clinical outcomes of HCC patients remains largely unknown.

In this study, using real-time quantitative PCR method, we measured ccf-mtDNA content in a hospitalbased cohort of HCC patients, and further evaluated the association of ccf-mtDNA content with clinical outcomes of HCC patients receiving TACE combined with TCM treatment.

\section{Material And Methods}

\section{Patients}

Informed consent was obtained from each patient according to the protocols approved by the ethics committees of the Affiliated Fifth People's Hospital of Ganzhou in Gannan Medical University. A total of 148 HBV-HCC patients were enrolled at the Department of Hepatology, Affiliated Fifth People's Hospital of Ganzhou in Gannan Medical University between January 2015 and October 2018. Each patient has two or more serum samples with different blood collection times, and the first serum was selected as the experimental sample after the patient was diagnosed with HCC. 7 patients who had been diagnosed with HCC before admission were excluded, and the final study included a total of 141 patients with HBV-HCC. In the subsequent statistical analysis of correlation of ccf-mtDNA content and prognosis, the study population was adjusted to 50 patients with HBV-HCC treated with TACE combined with TCM treatment, and the details were summarized in Figure 1.

\section{Serum Sampling}

Serum sample from each patient was collected in non-anticoagulated blood collection tubes, and then transferred to the laboratory on ice for processing within 6 hours. Serum samples were collected by twostep centrifugation: the serum sample was extracted from the whole blood after centrifuged with $1500 \mathrm{rpm}$ for $5 \mathrm{~min}$, and then remove the supernatant to a clean tube and re-centrifuged with $12000 \mathrm{rpm}$ for another $5 \mathrm{~min}$, the supernatant was collected into a $1.5 \mathrm{~mL}$ tube, and stored at $-80^{\circ} \mathrm{C}$ for use.

\section{Measurement of serum mtDNA content}


First, QIAamp DNA Blood Mini kit (Qiagen, Carlsbad, CA) was used to isolate the circulating DNA from $200 \mu \mathrm{L}$ serum sample according to the manufacturer's protocol, and mtDNA content was measured by

real-time qPCR with modified protocol described by previous studies ${ }^{15}{ }^{16}$. Briefly, the primer of ND1 gene was used for mtDNA amplification with the primer sequences as follows: ND1-F: 5'-

CCCTAAAACCCGCCACATCT-3' and ND1-R: 5'-GAGCGATGGTGAGAGCTAAGGT-3'. The primer of 36B4 gene was used as housekeeping gene control in all reaction, and the primer sequences were as follows: 36B4F: 5'-CAGCAAGTGGGAAGGTGTAATCC-3' and 36B4-R: 5'-CCCATTCTATCATCAACGGGTACAA-3'. The 20 $\mu \mathrm{L}$ qPCR reaction for ND1 and 36B4 consists of $1 X$ TB Green fast qPCR Mix (2X) (Takara), $0.2 \mu \mathrm{M}$ each primer, and $2 \mu \mathrm{L}$ of purified serum DNA sample. The thermal cycling conditions were at $95^{\circ} \mathrm{C}$ for $10 \mathrm{~min}$, followed by 40 cycles of $95^{\circ} \mathrm{C}$ for $15 \mathrm{~s}$ and $60^{\circ} \mathrm{C}$ for $60 \mathrm{~s}$ with signal acquisition. All samples were assayed in duplicate on a 96-well plate using QuantStudio ${ }^{\text {TM }} 7$ Flex qRT-PCR system (Applied Biosystems, USA). The same negative control and calibrator DNA samples were incorporated into each plate for quality control and calibration of PCR efficiency. A reference DNA sample was used to construct a standard curve for mtDNA measurement in each palate. For each standard curve, the reference DNA sample was serially diluted by $1: 3$ to produce a six-point standard curve. The $\mathrm{R}^{2}$ for each standard curve was $\geq 0.99$.

\section{Statistical analyses}

Use IBM SPSS 20.0 statistical software to analyze the collected clinical data and experimental data. Continuous variables are represented by average and standard deviation (mean \pm SD) or median and interquartile ranges according to specific types and categorical variables are represented by frequency and percentage. Cox proportional hazards regression model was used for univariate and multivariate analysis. Kaplan-Meier method was used to draw survival curves, and Log-Rank test and Breslow test were used for comparison between groups. The $\mathrm{P}<0.05$ was considered as the threshold of statistical significance.

\section{Results}

\section{Population characteristics of HBV-HCC patients}

In order to study the correlation between ccf-mtDNA content and the prognosis of HBV-HCC patients, the epidemiological and clinical characteristics of $141 \mathrm{HBV}-\mathrm{HCC}$ patients were analyzed. The results were shown in Table 1, the average age of the study population is $56.96 \pm 11.34$, the male to female ratio is about $5: 1$, and most of the patients were with no smoking (81.9\%), drinking (80.9\%), and family history of cancer (73\%). There were 45(31.9\%) patients developed liver cirrhosis, and 117 (83.0\%) patients were with B or C Child-Pugh classes. Among these 141 HBV-HCC patients, 70 (49.6\%) patients have been treated with TACE, 59 (41.8\%) patients have been treated with Chinese medicine, and 50 (35.4\%) of them have been treated with TACE combined with TCM. During the follow-up period, there were $88(62.4 \%)$ patients died, and the median survival time is 11 months (Inter-quartile range, 7.5-22 months) with the maximum survival is 44 months. 


\section{Correlation between ccf-mtDNA content and prognosis of HBV-HCC patients}

The median and tertile values of ccf-mtDNA content were used to group patients, and the correlation between ccf-mtDNA content and the prognosis of HBV-HCC patients was evaluated using univariate and multivariate analysis. As the data shown in Table 2, there is no statistical significance between the ccfmtDNA content and the overall survival of HBV-HCC patients in the univariate analysis. However, in the multivariate analyses, a bordline statistical significance association between ccf-mtDNA and HCC overall survival was observed in patients with higher ccf-mtDNA content by both median anaysis with HR of 1.49 $(95 \% \mathrm{Cl}, 0.94-2.34, p=0.09)$ and tertiel analysis with $\mathrm{HR}$ of $1.76(95 \% \mathrm{Cl}, 1.00-3.11, p=0.05)$ in third tertile group, comparing to patients with lower ccf-mtDNA, with adjusting for age, gender, drinking and smoking status, history of family cancer, liver cirrhosis, and TACE treatment. And the bordling significance effect of ccf-mtDNA content was remained in the tertile analysis with HR of $1.66(95 \% \mathrm{Cl}, 0.93-2.94, p=0.08)$ in the third tertile group, when further multivariate analysis with above variables plus adjuvant treatment with Chinese medicine.

The Kaplan-Meier analysis method is used to draw the survival curve of HBV-HCC patients with different ccf-mtDNA content (as shown in Figure 2). Results showed that there was no statistically significant difference in the overall survival rate between the low-content group and the high-content group of ccfmtDNA (Log Rank $p=0.732$ ).

\section{The correlation between ccf-mtDNA content and the prognosis of HBV-HCC patients receiving TACE combined with TCM treatment}

We further evaluate whether the ccf-mtDNA content correlate with the prognosis of HBV-HCC patients receiving TACE combined with TCM treatment. The epidemiological and clinical characteristics of 50 HBV-HCC patients with TACE combined with TCM treatment were analyzed. Results show (as shown in Table 3 ) the average age of the study population is $56.48 \pm 8.175$, the male to female ratio is about $6: 1$, and most of the patients were with no smoking (70\%), drinking (70\%), and family history of cancer (73\%). There were 34 (68\%) patients developed liver cirrhosis, and 36 (72\%) patients were with B or C Child-Pugh classes. During the follow-up period, there were $23(46 \%)$ patients died, and the median survival time is 20 months ( $95 \% \mathrm{Cl}: 12,29$ months), the maximum survival time is 44 months.

The patients were divided into groups by the median and tertile values of ccf-mtDNA content, and the correlation between ccf-mtDNA content and prognosis of HBV-HCC patients receiving TACE combined with TCM treatment was evaluated through univariate and multivariate analysis. It showed that the high content of ccf-mtDNA is an independent risk factor for the prognosis of HBV-HCC patients with TACE combined with TCM treatment (as shown in Table 4). And in the multivariate analysis, a significance association between the ccf-mtDNA content and the overall survival of HBV-HCC patients were observed in patients with high content by median analysis with $\mathrm{HR}$ of $4.010(95 \% \mathrm{Cl}, 1.252-12.844, \mathrm{p}=0.02)$ and tertile analysis with HR $4.554(95 \% \mathrm{Cl}, 1.159-17.811, \mathrm{P}=0.03), 5.322(95 \% \mathrm{Cl}, 1.053-26.909, \mathrm{p}=0.04)$ in second and third tertile group, comparing to patients with lower ccf-mtDNA, with adjusting for gender, 
age, cirrhosis, liver function, history of drinking, smoking history, family cancer history, tumor number, tumor size.

The Kaplan-Meier analysis method is used to draw the survival curve of HBV-HCC patients receiving TACE combined with TCM treatment according to the content of ccf-mtDNA (as shown in Figure 3A). Results showed the prognosis of patients with high content of ccf-mtDNA was worse, but the difference in overall survival rate between the two groups is not statistically significant ( $\log R a n k P=0.097$ ). Further analysis found that 3 patients in the study died less than six months after admission, and this may affect the accuracy of the survival analysis results. The analysis was repeated after excluding these 3 abnormal samples, and results showed that (as shown in Figure 3B) the prognosis of the patients in the group with high content of ccf-mtDNA content was worse, and the overall survival rate difference between the two groups was statistically significant (Log Rank $\mathrm{P}=0.027$ ).

\section{Discussion}

In this retrospective study, we measured ccf-mtDNA content in $\mathrm{HCC}$ and demonstrate that patients with low ccf-mtDNA content have increased benefit from TACE combined with TCM treatment. In the multivariate analysis of $50 \mathrm{HBV}-\mathrm{HCC}$ patients, the results obtained by the median and tertile analysis showed that the prognosis of patients with different ccf-mtDNA content was significantly different, and both were statistically significant with $p$ value of 0.02 by median analysis and $p$ value of 0.04 in third group by tertile analysis. Our data indicated that the high content of ccf-mtDNA is an independent risk factor for the prognosis of HBV-HCC patients with TACE combined with TCM treatment. Kaplan-Meier survival analysis after excluding 3 abnormal samples showed that the prognosis of patients in the group with high content of ccf-mtDNA content was worse, and the difference in overall survival rate between the two groups was statistically significant (Log Rank $P=0.027)$.

Circulating cell-free mitochondrial DNA (ccf-mtDNA) is that mtDNA fragments are released outside the cell and into the circulation by cell necrosis and secretion. In the last decade, the roles ccf-mtDNA as potential noninvasive biomarkers have been demonstrated in numerous different types of disease, including cancer. Several studies ${ }^{13}, 17,18$ show that ccf-mtDNA in plasma or serum could be used as a new and effective diagnostic and prognostic marker in many solid tumors. Ellinger's research showed that the ccf-mtDNA content in the serum of patients with urinary system malignancies increased significantly ${ }^{17}$, and Mahmoud's research showed that ccf-mtDNA has a good prognostic value in breast cancer $^{18}$. Meng et al. reported that increasing levels of $c$ cf mtDNA has a significant association with epithelial ovarian cancer progress and poor prognosis ${ }^{19}$. These studies suggested that ccf-mtDNA is a potential tumor molecular marker, and further studies are needed to reveal the mechanism and clinical value of changes in ccf-mtDNA content.

Transarterial chemoembolization (TACE) is the firstline treatment for intermediate stage disease, which includes asymptomatic patients with limited unresectable multinodular lesions, without vascular invasion or extrahepatic spread and who have well-preserved liver function ${ }^{20}$, although its clinical effect is still far 
from satisfactory. Meta-analysis showed Traditional Chinese Medicine (TCM) improves immune response for unresectable hepatocellular carcinoma (UHCC) after transcatheter arterial chemoembolization (TACE) ${ }^{21}$. TCM is gathering increasing interest due to the immunoregulatory properties of certain compounds, it can restore immunosurveillance in HCC to promote anti-tumor effects in several ways, including the upregulation of immunostimulatory factors and the downregulation of immunosuppressive factors ${ }^{22}$. And also clinical studies have demonstrated the utility of TCM in relieving adverse events after TACE, Tang et al. found the chinese medicine Jianpi Ligan decoction (JLD) was effective for reduction of side effects and improvement of long-term survival for patients with unresectable HCC treated by TACE ${ }^{23}$. However, the cellular and molecular mechanisms of TCM-mediated anti-tumor effect is still not clear, and effective predictive biomarkers are needed to increase efficacy and survival for HCC patients receiving TACE combine with TCM treatment.

Due to the limitation of sample size in this study, some results can't fully reflect the role of ccf-mtDNA content in the prognosis of HBV-HCC patients with TACE combined with TCM treatment. Therefore, further researches are needed to verify these finding with large number of samples. In summary, this study showed that the ccf-mtDNA content in serum has a certain value for the prognosis assessment of HBV-HCC patients receiving TACE combined with TCM treatment, and it may be used as a potential molecular marker for the prognosis of HCC patients.

\section{Abbreviations}

HCC: Hepatocellular carcinoma; HBV: Hepatitis B virus; TACE: trans-hepatic arterial chemoembolization; TCM: traditional Chinese medicine; BCLC: Barcelona clinic liver cancer; mtDNA: The circular genome of mitochondrial; ccf-mtDNA: circulating cell-free mtDNA; JLD: Jianpi Ligan decoction;

\section{Declarations}

\section{Ethics approval and consent to participate}

Not applicable

Consent for publication

Not applicable

\section{Availability of data and materials}

Not applicable

\section{Competing interests}

The authors declare that they have no competing interests. 


\section{Funding}

National Natural Science Foundation of China (No.82060524) and Science and Technology Innovation Outstanding Young Talents Training Program of Jiangxi Province (20192BCBL23017)

\section{Authors' contributions}

SW and GZ conceived and designed the experiment, GZ and JX wrote the manuscript, YL, SL and HL performed the experiments, FX, XL and QZ collected the samples and clinical data, SW and GZ analyzed the data. All authors are in agreement with the content of the manuscript and this submission.

\section{Acknowledgement}

This work was supported by the National Natural Science Foundation of China (No.82060524), Science and Technology Innovation Outstanding Young Talents Training Program of Jiangxi Province (20192BCBL23017).

\section{References}

[1] Xie Y. Hepatitis B Virus-Associated Hepatocellular Carcinoma. ADV EXP MED BIOL. 2017; 1018: 11-21.

[2][Strategies of primary prevention of liver cancer in China: expert consensus (2018)]. Zhonghua Yu Fang Yi Xue Za Zhi. 2019; 53: 36-44.

[3] Gomaa Al. Recent advances in multidisciplinary management of hepatocellular carcinoma. World Journal of Hepatology. 2015; 7: 673.

[4] Chen Q, Wu P, Huang T, Shen L, Huang Z, Li W. Efficacy of treatment regimens for advanced hepatocellular carcinoma. MEDICINE. 2019; 98: e17460.

[5] Veltri KL, Espiritu M, Singh G. Distinct genomic copy number in mitochondria of different mammalian organs. J CELL PHYSIOL. 1990; 143: 160-4.

[6] Xiong Y, Lu QJ, Zhao J, Wu GY. Metformin inhibits growth of hepatocellular carcinoma cells by inducing apoptosis via mitochondrion-mediated pathway. Asian Pac J Cancer Prev. 2012; 13: 3275-9.

[7] Dilip A, Cheng G, Joseph J, et al.. Mitochondria-targeted antioxidant and glycolysis inhibition: synergistic therapy in hepatocellular carcinoma. Anticancer Drugs. 2013; 24: 881-8.

[8] Wenceslau CF, McCarthy CG, Szasz T, Spitler K, Goulopoulou S, Webb RC. Mitochondrial damageassociated molecular patterns and vascular function. EUR HEART J. 2014; 35: 1172-7.

[9] Reznik E, Miller ML, Senbabaoglu Y, et al.. Mitochondrial DNA copy number variation across human cancers. ELIFE. 2016; 5. 
[10] Fliss MS, Usadel H, Caballero OL, et al.. Facile detection of mitochondrial DNA mutations in tumors and bodily fluids. SCIENCE. 2000; 287: 2017-9.

[11] Bao D, Ba Y, Zhou F, et al.. Alterations of telomere length and mtDNA copy number are associated with overall survival in hepatocellular carcinoma patients treated with transarterial chemoembolization. CANCER CHEMOTH PHARM. 2016; 78: 791-9.

[12] Yu M. Circulating cell-free mitochondrial DNA as a novel cancer biomarker: opportunities and challenges. MITOCHONDR DNA. 2012; 23: 329-32.

[13] Li L, Hann H, Wan S, et al.. Cell-free circulating mitochondrial DNA content and risk of hepatocellular carcinoma in patients with chronic HBV infection. SCI REP-UK. 2016; 6.

[14] Weerts M, Timmermans EC, van de Stolpe A, et al.. Tumor-Specific Mitochondrial DNA Variants Are Rarely Detected in Cell-Free DNA. NEOPLASIA. 2018; 20: 687-96.

[15] Fu X, Wan S, Hann HW, et al.. Relative telomere length: a novel non-invasive biomarker for the risk of non-cirrhotic hepatocellular carcinoma in patients with chronic hepatitis $\mathrm{B}$ infection. EUR J CANCER. 2012; 48: 1014-22.

[16] Wan S, Hann HW, Myers RE, et al.. Telomere length in circulating serum DNA as a novel non-invasive biomarker for cirrhosis: a nested case-control analysis. LIVER INT. 2012; 32: 1233-41.

[17] Ellinger J, Muller DC, Muller SC, et al.. Circulating mitochondrial DNA in serum: a universal diagnostic biomarker for patients with urological malignancies. Urol Oncol. 2012; 30: 509-15.

[18] Mahmoud EH, Fawzy A, Ahmad OK, Ali AM. Plasma Circulating Cell-free Nuclear and Mitochondrial DNA as Potential Biomarkers in the Peripheral Blood of Breast Cancer Patients. Asian Pac J Cancer Prev. 2015; 16: 8299-305.

[19] Meng $X$, Schwarzenbach $\mathrm{H}$, Yang $\mathrm{Y}$, et al.. Circulating Mitochondrial DNA is Linked to Progression and Prognosis of Epithelial Ovarian Cancer. TRANSL ONCOL. 2019; 12: 1213-20.

[20] Raoul J, Forner A, Bolondi L, Cheung TT, Kloeckner R, de Baere T. Updated use of TACE for hepatocellular carcinoma treatment: How and when to use it based on clinical evidence. CANCER TREAT REV. 2019; 72: 28-36.

[21] Meng M, Wen Q, Cui Y, She B, Zhang R. Meta-analysis: Traditional Chinese Medicine for Improving Immune Response in Patients With Unresectable Hepatocellular Carcinoma After Transcatheter Arterial Chemoembolization. EXPLORE. 2011; 7: 37-43.

[22] Jia W, Wang L. Using Traditional Chinese Medicine to Treat Hepatocellular Carcinoma by Targeting Tumor Immunity. Evid Based Complement Alternat Med. 2020; 2020: 9843486. 
[23] Tang CW, Zhu M, Feng WM, Bao Y, Zheng YY. Chinese herbal medicine, Jianpi Ligan decoction, improves prognosis of unresectable hepatocellular carcinoma after transarterial chemoembolization: a retrospective study. Drug Des Devel Ther. 2016; 10: 2461-6.

\section{Tables}

Table 1. The characteristics of study population 


\begin{tabular}{|c|c|}
\hline Characteristics & Number $\rrbracket n=141 \rrbracket \% \rrbracket$ \\
\hline \multicolumn{2}{|l|}{ Age } \\
\hline$\leq 57$ & $71 \otimes 50.4 \rrbracket$ \\
\hline$\varangle 57$ & $70 \rrbracket 49.6 \rrbracket$ \\
\hline \multicolumn{2}{|l|}{ Gender } \\
\hline Male & $118 \rrbracket 83.7 \rrbracket$ \\
\hline Female & $23 \rrbracket 16.3 \rrbracket$ \\
\hline \multicolumn{2}{|l|}{ Smoking } \\
\hline No & 114『81.9凶 \\
\hline Yes & 27ه19.1】 \\
\hline \multicolumn{2}{|l|}{ Drinking } \\
\hline No & $114 \rrbracket 80.9 \rrbracket$ \\
\hline Yes & 27ه19.1ه \\
\hline \multicolumn{2}{|c|}{ Family history of cancer } \\
\hline No & 103囚73.0® \\
\hline Yes & $38 \rrbracket 27.0 \rrbracket$ \\
\hline \multicolumn{2}{|l|}{ Liver cirrhosis } \\
\hline No & $96 \bowtie 68.1 \rrbracket$ \\
\hline Yes & $45 \rrbracket 31.9 \rrbracket$ \\
\hline \multicolumn{2}{|l|}{ Child-Pugh class } \\
\hline$A$ & $24 \rrbracket 17.0 \rrbracket$ \\
\hline B & $69 \rrbracket 48.9 \rrbracket$ \\
\hline C & $48 \rrbracket 34.1 \rrbracket$ \\
\hline \multicolumn{2}{|l|}{ TACE treatment } \\
\hline No & $71 \rrbracket 50.4 \rrbracket$ \\
\hline Yes & $70 \rrbracket 49.6 \rrbracket$ \\
\hline \multicolumn{2}{|c|}{ TCM adjuvant treatment } \\
\hline No & $82(58.2)$ \\
\hline Yes & $59(41.8)$ \\
\hline
\end{tabular}

Page $11 / 17$ 


\begin{tabular}{|ll|}
\hline \multicolumn{1}{|l|}{ Vital status } & \\
\hline \multicolumn{1}{|c|}{ Dead } & $88(62.4)$ \\
\hline Live & $53(37.6)$ \\
\hline Survival time (Month) (median, quartile range) & $11.0(7.5-22.0)$ \\
ccf-mtDNA content (median, quartile range) & $1.98(1.17-3.68)$ \\
\hline
\end{tabular}

Table 2. The association between ccf-mtDNA and overall survival of HBV-HCC patients

\begin{tabular}{|c|c|c|c|c|c|c|c|c|}
\hline \multirow{2}{*}{$\begin{array}{l}\text { ccf- } \\
\text { mtDNA }\end{array}$} & \multirow{2}{*}{$\begin{array}{l}\text { Number of } \\
\text { patient }\end{array}$} & \multirow{2}{*}{$\begin{array}{l}\text { Number } \\
\text { of death }\end{array}$} & \multicolumn{2}{|c|}{ Univariate } & \multicolumn{2}{|c|}{ Multivariate $^{\dagger}$} & \multicolumn{2}{|c|}{ Multivariate ${ }^{\ddagger}$} \\
\hline & & & $\begin{array}{l}\mathrm{HR} \\
\text { ख95\%Cl囚 }\end{array}$ & $\begin{array}{l}P \\
\text { value }\end{array}$ & $\begin{array}{l}\mathrm{HR} \\
\text { \95\%Cl }\end{array}$ & $\begin{array}{l}P \\
\text { value }\end{array}$ & $\begin{array}{l}\mathrm{HR} \\
\text { \95\%Cl囚 }\end{array}$ & $\begin{array}{l}P \\
\text { value }\end{array}$ \\
\hline \multicolumn{9}{|l|}{$\begin{array}{l}\text { By } \\
\text { median }\end{array}$} \\
\hline Lower & 71 & 45 & 1.00 & & 1.00 & & 1.00 & \\
\hline Higher & 71 & 43 & $\begin{array}{l}0.93 \\
\rrbracket 0.86- \\
4.74 \rrbracket\end{array}$ & 0.74 & $\begin{array}{l}1.49 \\
\rrbracket 0.94- \\
2.34 \rrbracket\end{array}$ & 0.09 & $\begin{array}{l}1.44 \\
\otimes 0.91- \\
2.29 \rrbracket\end{array}$ & 0.12 \\
\hline \multicolumn{9}{|l|}{$\begin{array}{l}\text { By } \\
\text { tertile }\end{array}$} \\
\hline $\begin{array}{c}1^{\text {st }} \\
\text { tertile }\end{array}$ & 47 & 28 & 1.00 & & 1.00 & & 1.00 & \\
\hline $\begin{array}{l}2^{\text {nd }} \\
\text { tertile }\end{array}$ & 47 & 32 & $\begin{array}{l}1.45 \\
\rrbracket 0.87- \\
2.41 \rrbracket\end{array}$ & 0.16 & $\begin{array}{l}1.58 \\
₫ 0.89- \\
2.80 \rrbracket\end{array}$ & 0.12 & $\begin{array}{l}1.60 \\
\rrbracket 0.90- \\
2.84 \rrbracket\end{array}$ & 0.11 \\
\hline $\begin{array}{l}3^{\text {rd }} \\
\text { tertile }\end{array}$ & 47 & 28 & $\begin{array}{l}1.18 \\
\otimes 0.70- \\
1.99 \square\end{array}$ & 0.55 & $\begin{array}{l}1.76 \\
\otimes 1.00- \\
3.11 \rrbracket\end{array}$ & 0.05 & $\begin{array}{l}1.66 \\
\rrbracket 0.93- \\
2.94 \rrbracket\end{array}$ & 0.08 \\
\hline $\begin{array}{l}P \text { for } \\
\text { trend }\end{array}$ & & & & 0.36 & & 0.13 & & 0.17 \\
\hline \multicolumn{9}{|c|}{$\begin{array}{l}\text { Notes: }{ }^{\dagger} \text { Multivariate analysis adjusted for age, gender, drinking and smoking status, history of family } \\
\text { cancer, liver cirrhosis, and TACE treatment. }{ }^{\ddagger} \text { Multivariate analysis adjusted for age, gender, drinking } \\
\text { and smoking status, history of family cancer, liver cirrhosis, TACE treatment, and adjuvant treatment } \\
\text { with Chinese medicine. }\end{array}$} \\
\hline
\end{tabular}

Table 3. The characteristics of subpopulation with TACE and TCM treatments 


\begin{tabular}{|c|c|}
\hline Characteristics & Number $\ n=50, \% \rrbracket$ \\
\hline \multicolumn{2}{|l|}{ Age } \\
\hline$\leq 55$ & $25 \rrbracket 50.0 \otimes$ \\
\hline$\nabla 55$ & $25 \rrbracket 50.0 \otimes$ \\
\hline \multicolumn{2}{|l|}{ Gender } \\
\hline Male & $43 \llbracket 86.0 \otimes$ \\
\hline Female & $7 \rrbracket 14.0 \bigotimes$ \\
\hline \multicolumn{2}{|l|}{ Smoking status } \\
\hline No & $35 \bigotimes 70.0 \rrbracket$ \\
\hline Yes & $15 \rrbracket 30.0 \rrbracket$ \\
\hline \multicolumn{2}{|l|}{ Drinking status } \\
\hline No & $35 \rrbracket 70.0 \otimes$ \\
\hline Yes & $15 \rrbracket 30.0 \rrbracket$ \\
\hline \multicolumn{2}{|c|}{ Family history of cancer } \\
\hline No & $33 \rrbracket 66.0 \bigotimes$ \\
\hline Yes & $17 \rrbracket 34.0 \rrbracket$ \\
\hline \multicolumn{2}{|l|}{ Liver cirrhosis } \\
\hline No & $34 \llbracket 68.0 \rrbracket$ \\
\hline Yes & 16囚32.0区 \\
\hline \multicolumn{2}{|c|}{ Child-pugh classification } \\
\hline A & $14 \llbracket 28.0 \rrbracket$ \\
\hline B & $26 \rrbracket 52.0 \rrbracket$ \\
\hline C & $10 \otimes 20.0 \rrbracket$ \\
\hline \multicolumn{2}{|l|}{ Tumor number } \\
\hline Single & $32 \bowtie 64.0 \rrbracket$ \\
\hline Multiple & $18 \rrbracket 36.0 \rrbracket$ \\
\hline \multicolumn{2}{|l|}{ Tumor Size } \\
\hline$\leq 5$ & $27 \rrbracket 54.0 \rrbracket$ \\
\hline$>5$ & $23 \rrbracket 46.0 \rrbracket$ \\
\hline
\end{tabular}

Page 13/17 


\begin{tabular}{|ll|}
\hline \multicolumn{1}{|l|}{ Vital status } & \\
\hline \multicolumn{1}{|c|}{ Death } & $23 \varangle 46.0 \rrbracket$ \\
\hline Live & $27 \otimes 54.0 \rrbracket$ \\
\hline Survival time (Month) (median, quartile range) & $20.0(12.0-29.0)$ \\
\hline ccf-mtDNA content (median, quartile range) & $1.58(0.77-3.27)$ \\
\hline
\end{tabular}

Table 4. The asociation between ccf-mtDNA and prognosis of HBV-HCC patients with TACE and TCM treatments

\begin{tabular}{|c|c|c|c|c|c|c|}
\hline \multirow{2}{*}{$\begin{array}{l}\text { ccf- } \\
\text { mtDNA }\end{array}$} & \multirow{2}{*}{$\begin{array}{l}\text { Number of } \\
\text { patient }\end{array}$} & \multirow{2}{*}{$\begin{array}{l}\text { Number of } \\
\text { death }\end{array}$} & \multicolumn{2}{|l|}{ Univariate } & \multicolumn{2}{|c|}{ Multivariate $^{\dagger}$} \\
\hline & & & $\mathrm{HR} \rrbracket 95 \% \mathrm{Cl} \nabla$ & $\begin{array}{l}P \\
\text { value }\end{array}$ & $\mathrm{HR} \otimes 95 \% \mathrm{Cl} \nabla$ & $\begin{array}{l}P \\
\text { value }\end{array}$ \\
\hline \multicolumn{7}{|l|}{$\begin{array}{l}\text { By } \\
\text { median }\end{array}$} \\
\hline Lower & 25 & 9 & 1.00 & & 1.00 & \\
\hline Higher & 25 & 14 & $\begin{array}{l}2.02 \rrbracket 0.86- \\
4.74 \rrbracket\end{array}$ & 0.11 & $\begin{array}{l}4.0101 .25- \\
12.84 \square\end{array}$ & 0.02 \\
\hline \multicolumn{7}{|l|}{ By tertile } \\
\hline $\begin{array}{c}1^{\text {st }} \\
\text { tertile }\end{array}$ & 17 & 6 & 1.00 & & 1.00 & \\
\hline $\begin{array}{l}2^{\text {nd }} \\
\text { tertile }\end{array}$ & 17 & 10 & $\begin{array}{l}2.66 \rrbracket 0.94- \\
7.52 \rrbracket\end{array}$ & 0.07 & $\begin{array}{l}4.5501 .16- \\
17.810\end{array}$ & 0.03 \\
\hline $\begin{array}{c}3^{\text {rd }} \\
\text { tertile }\end{array}$ & 16 & 7 & $\begin{array}{l}1.60 \rrbracket 0.53- \\
4.85 \rrbracket\end{array}$ & 0.40 & $\begin{array}{l}5.3201 .05- \\
26.910\end{array}$ & 0.04 \\
\hline $\begin{array}{l}P \text { for } \\
\text { trend }\end{array}$ & & & & 0.17 & & 0.07 \\
\hline
\end{tabular}

\section{Figures}




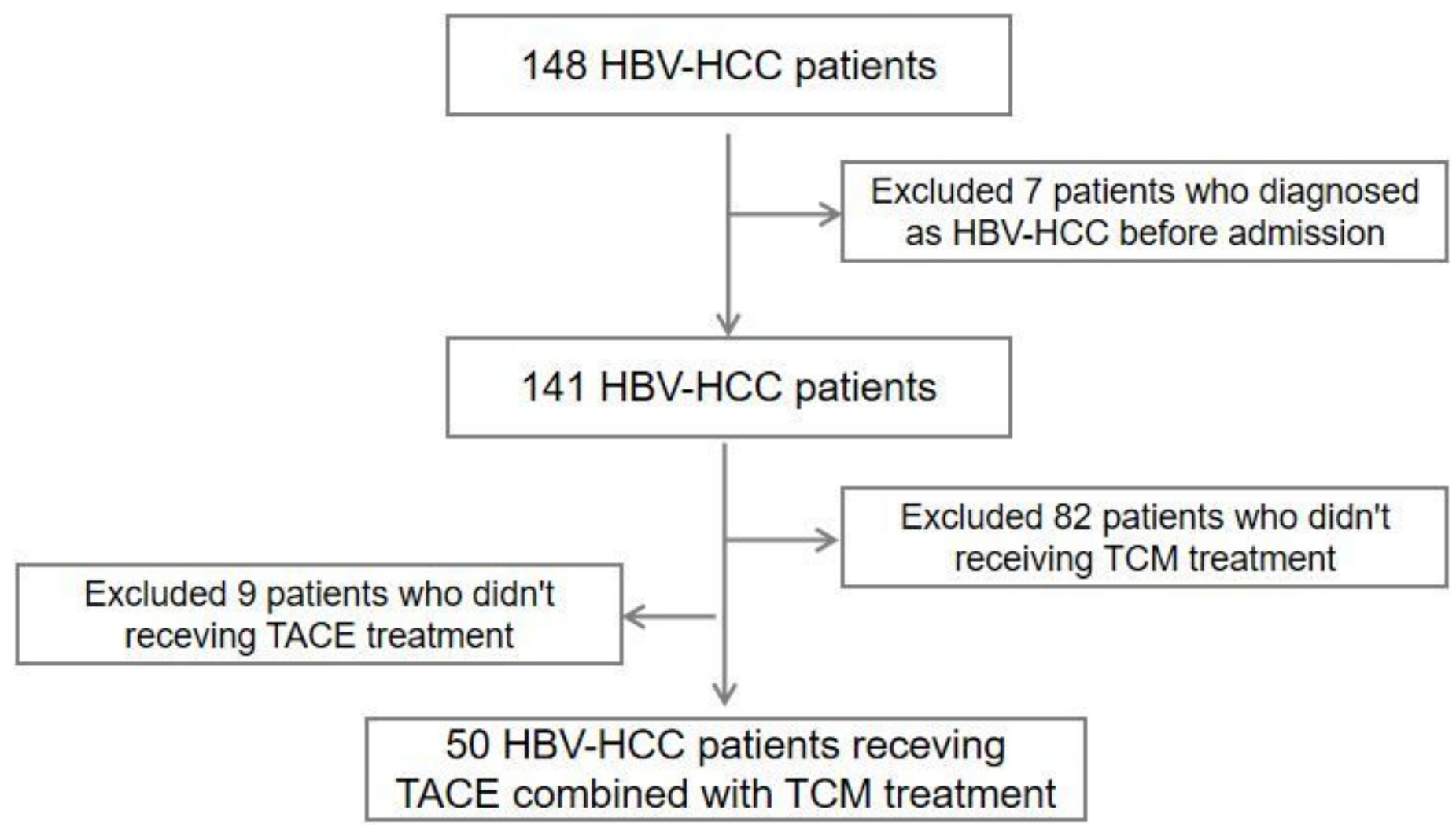

Figure 1

The flowchart of patient enrollment. 


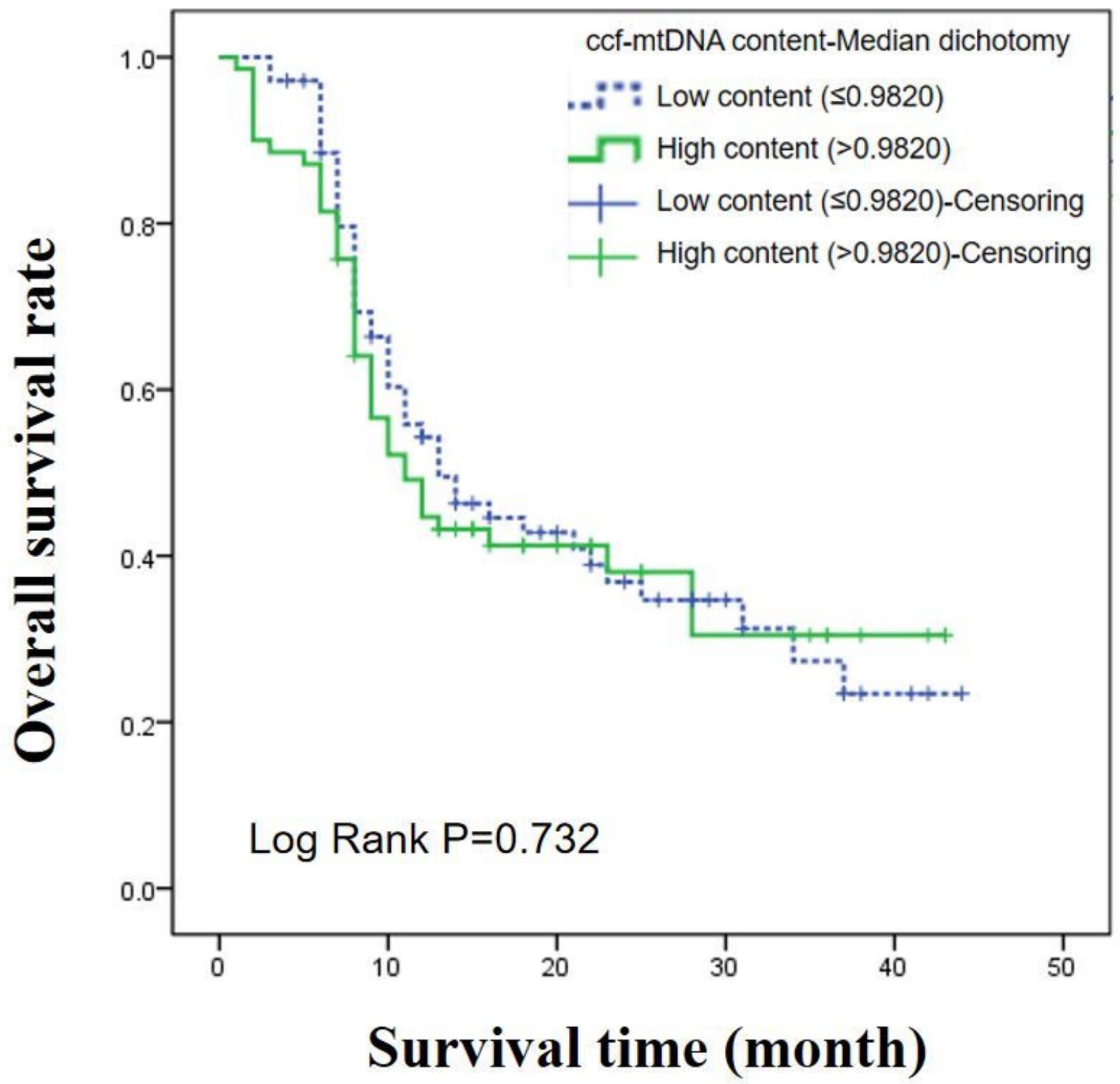

Figure 2

Kaplan-Meier curve analysis of ccf-mtDNA content and HBV-HCC patients 
A

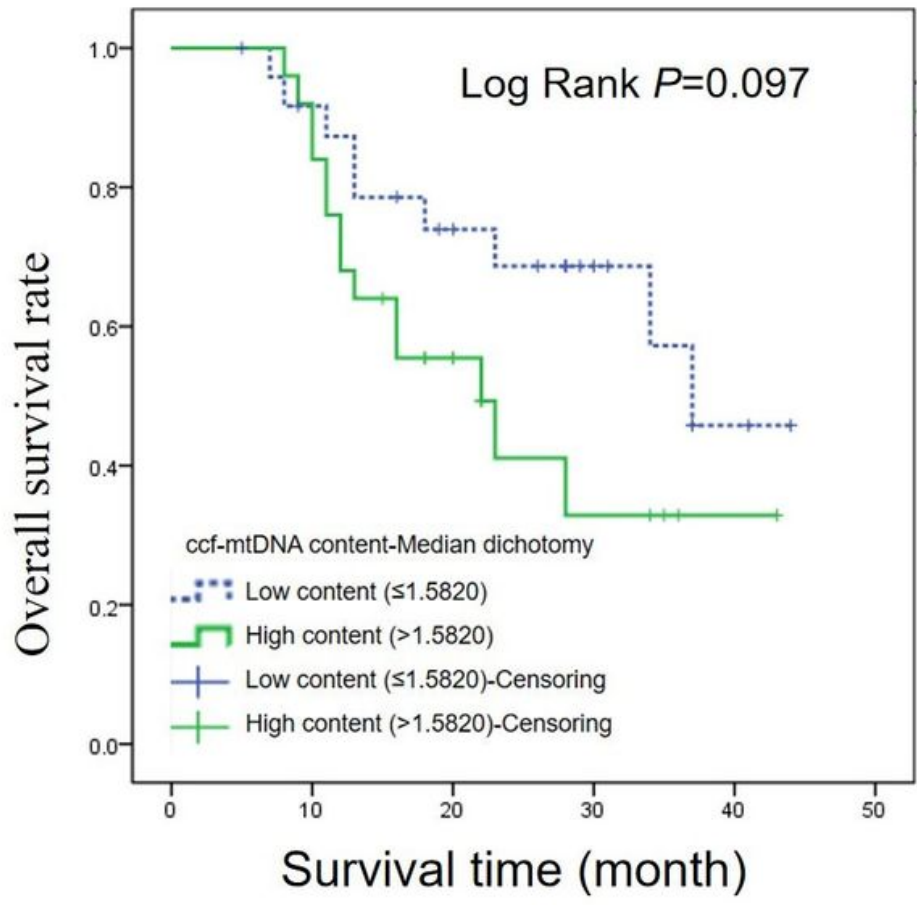

B

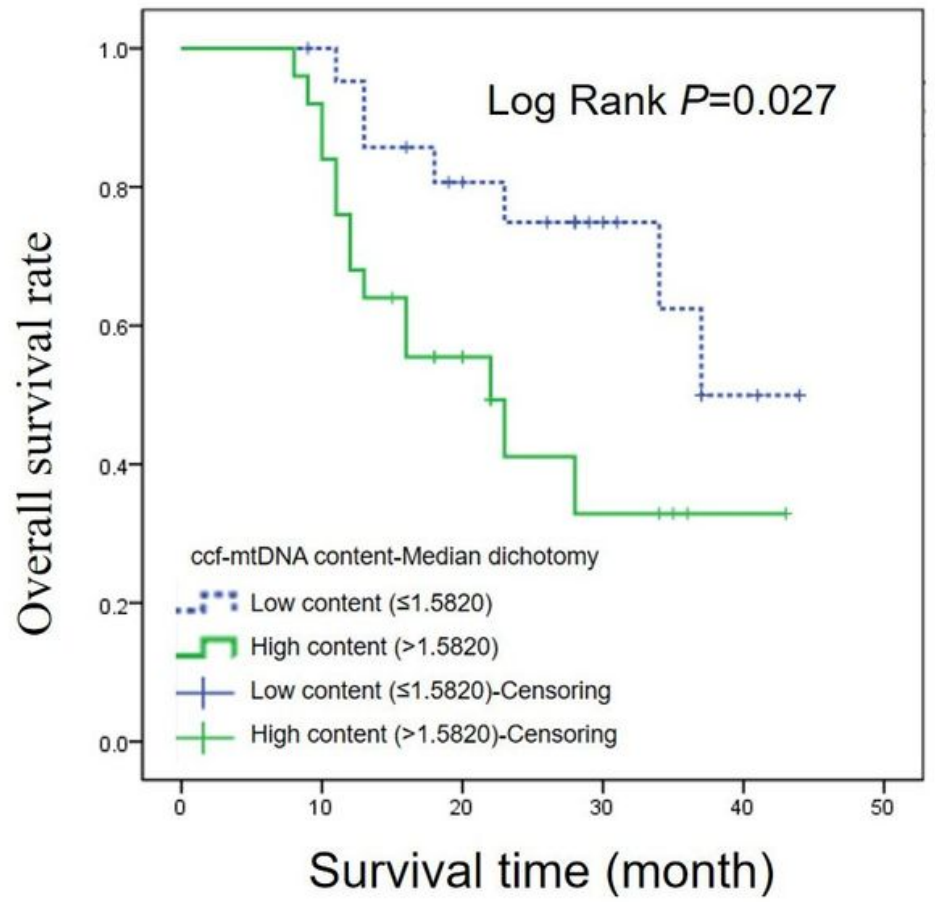

\section{Figure 3}

Kaplan-Meier curve analysis of ccf-mtDNA content and HBV-HCC patients receiving TACE combined with TCM treatment. Panel A included all HBV-HCC patients $(n=50)$ treated with TACE and TCM, panel B included patients $(n=47)$ with excluding 3 patients died less than six months after admission. 